\title{
A Unifying Theory of Aging between Modern Medicine and Traditional Chinese Medicine
}

\author{
Kai-Chit Cheung, Hoi-Yan Leung, Kam-Ming Ko* \\ Division of Life Science, Hong Kong University of Science \& Technology, Hong Kong, China \\ Email: ^bcrko@ust.hk
}

How to cite this paper: Cheung, K.-C., Leung, H.-Y. and Ko, K.-M. (2020) A Unifying Theory of Aging between Modern Medicine and Traditional Chinese Medicine. Chinese Medicine, 11, 105-112. https://doi.org/10.4236/cm.2020.112006

Received: May 18, 2020

Accepted: June 13, 2020

Published: June 16, 2020

Copyright $\odot 2020$ by author(s) and Scientific Research Publishing Inc. This work is licensed under the Creative Commons Attribution International License (CC BY 4.0).

http://creativecommons.org/licenses/by/4.0/

(c) (i) Open Access

\begin{abstract}
With the increasing aging population around the world as a result of birth rates and advances in medical technologies, there is an urgent need to unravel the primary cause of aging, in the hope of developing a rational approach to retard the aging process. This is crucial to reduce the societal impact of aging. Although modern medicine and traditional Chinese medicine view the process of aging from different perspectives, this article aims to develop a common understanding between these two distinct medical systems in relation to the aging process. As such, a unified approach can hopefully be developed to effectively slow down the aging process. Modern medicine has proposed the "mitochondrial theory" of aging, which implicates a causal relationship between the rate of mitochondrial reactive oxygen species generation and lifespan as noted in a wide spectrum of primate species, while traditional Chinese medicine views aging as the result of a gradual depletion of congenital primordial Qi, which can be spared by the sufficient generation of postnatal pectoral Qi. By amalgamating the knowledge of modern medicine with that of traditional Chinese medicine in relation to aging, it is suggested that while Chinese tonifying herbs can be used to restore the balance of body functions enabling the generation of sufficient pectoral Qi, active ingredients isolated from Chinese tonifying herbs or other plant sources capable of inducing mitohormesis and mitophagy, as well as senolysis, can be used to retard the aging process.
\end{abstract}

\section{Keywords}

Aging Theory, Modern Medicine, Chinese Medicine, Mitochondria

\section{Introduction}

The increasing aging population remains an impending problem in modern society. This increasing number of the elderly in the population will not only result 
in a much greater proportion of health care services needed for the management of aging-associated degenerative diseases, but the increasing elderly dependency ratio (i.e. the number of elderly persons aged 65 or above raised by every 1000 employed persons) would also strain the labor force and pose a financial burden to governments. Therefore, identifying the primary cause of aging and efforts to develop effective approaches to retarding the inevitable process of aging has become an area of considerable interest in biomedical research and societal health.

To unify the concept of aging in relation to both modern medicine and traditional Chinese medicine, reliable theories and potential interventions to slow down the process of aging may be discovered. The "mitochondrial theory" of aging in modern medicine is, by far, more viable than other theories of aging, such as endocrine, immunological, telomere, or other theories [1]. Reactive oxygen species (ROS) generated from mitochondria is considered to be a primary cause of aging because this appears to correlate with differences in lifespan across a wide spectrum of primate species. Accordingly, several approaches to reducing the rate of mitochondrial ROS production (mtROSp) or safeguarding mitochondrial function have been proposed, including dietary restriction and the induction of mitophagy or mitohormesis. On the other hand, traditional Chinese medicine theory emphasizes the importance of the balance of organ functions in terms of Yin, Yang, Qi, and Blood, in retarding the aging process [2]. A more effective and feasible approach in the prevention of age-associated diseases and slowing the process of aging can hopefully be developed by the combination of traditional Chinese medicine and modern medicine approaches.

\section{Mitochondrial Theory of Aging}

As a result of almost half a century of scientific debate in modern medicine, it is now generally accepted that the primary cause of aging is the rate of mtROSp [3]. During the aging process, the increase in the production of ROS, which is inevitably caused by mitochondrial electron transport chain activity during ATP generation, is known to impose oxidative stress on mitochondria and other cellular organelles, resulting in the oxidation of membrane lipids and other macromolecules such as DNA, causing the disruption of cellular and structural integrity, and ultimately cell death. This aging hypothesis is consistent with the observation that there is a negative correlation between the rate of mtROSp and lifespan across species, i.e. long-lived mammals and birds typically have lower values of mtROSp, and vice versa [3].

Oxidative stress within mitochondria can have a detrimental effect on nuclear DNA, contributing to aging. DNA damage, manifesting as severe double-strand breaks, can occur within mitochondrial DNA (mtDNA), which may result in impaired function of mtDNA-encoded electron transport chain or mitochondrial ribosome subunits in a certain tissue [3] [4]. However, what drives the process of aging more directly is the generation of mtDNA fragments from ROS-induced double-strand breakage, which can insert themselves into the nuclear DNA. Stu- 
dies in yeast and rat livers and brains have demonstrated that mtDNA fragments accumulate within nuclear DNA with increasing age [5]. As these mtDNA fragments migrate from mitochondria to the nuclear DNA, they can accumulate at the pericentromeric regions of chromosomes, likely producing a marked interference of the mtDNA fragments with mitotic components [3] [6]. The resultant nuclear genome is susceptible to aneuploidy, which is defined by an abnormal number of chromosomes in a cell, and genetic instability. The insertion of mtDNA into nuclear DNA can, therefore, lead to mitotic decline and aneuploidy-driven senescence in cells [6].

\section{Modern Medical Approaches to Delaying the Aging Process}

Based on the aforementioned "mitochondrial theory" of aging, several modern medicine approaches to delaying the aging process and extend the lifespan and healthspan of humans have been proposed. Experimental studies have shown a correlation between the restriction of caloric intake (or even protein intake) and the decrease in the rate of mtROSp. Studies using rats and mice have shown that protein restriction results in an increase in lifespan, which accounts for about $50 \%$ of the life-prolonging effect of caloric restriction [7]. More specifically, the substitution of L-methionine in the diet with L-glutamate achieving an $80 \%$ methionine restriction was found to decrease the rate of mtROSp in mice. While the biochemical mechanism underlying this experimental observation remains unclear, adopting caloric or protein restriction would seem to be an effective method for reducing the rate of mtROSp and hence the oxidative stress in mitochondria and cells generally.

Given that mitochondria play a crucial role in the aging process, interventions that preserve the integrity of mitochondrial function should be effective in retarding the aging process. Autophagy, which is a cellular process involving a degradation system that delivers certain targeted materials to lysosomes for degradation, is found to be an important determinant of the longevity of animals, as evidenced by the observation that long-lived animals typically show elevated levels of autophagic activity [8]. Mitophagy, selective degradation of mitochondria by autophagy, is responsible for removing dysfunctional mitochondria and safeguarding overall mitochondrial function [9]. Given the beneficial effect of autophagy and mitophagy in maintaining cellular and mitochondrial function, therapeutic interventions that activate autophagy and/or mitophagy may provide a promising approach to delaying the process of aging.

Another approach to maintaining mitochondrial function is the activation of mitohormesis. Mitohormesis is a response to mitochondrial oxidative stress induced by ROS and other mitochondrial metabolites [10]. The activation of mitohormesis has been found to increase lifespan as well as healthspan in various animal models, with the latter being associated with improved metabolic status and immune function. When cells are exposed to sub-lethal levels of mitochondrial oxidative stress, a retrograde signaling pathway involving nuclear gene ex- 
pression is activated. The altered gene expression elicits adaptive responses including biogenesis of mitochondria and increased antioxidant defenses that can lead to the extension of lifespan [11]. Therefore, the activation of mitohormesis is vital in safeguarding mitochondrial and cellular structural and functional integrity.

One phenomenon associated with the process of aging is the presence of an increased number of senescent cells in tissues [12]. DNA damage, telomere shortening, or exposure to ROS are possible causes of cellular senescence, which potentially threatens the functional integrity of neighbouring normal cells by secreting high levels of inflammatory cytokines or immune modulators. One recent approach to slowing down aging involves the use of senolytic drugs that can induce apoptosis specifically in senescent cells (these being otherwise resistant to apoptosis). Senolytic drugs, such as dasatinib, quercetin and fisetin, have been found to reduce the number of senescent cells in mice and humans, thereby protecting normal cells against the inflammatory stress imposed by senescent cells [13] [14]. The use of senolytic agents to deal with the aging-related cellular senescence could provide an alternative approach to retarding the aging process.

Apart from the aforementioned approach in retarding the aging process, there is an on-going debate on whether or not the current approach of dietary antioxidant intake can effectively increase lifespan and slow down aging. Theoretically, taking exogenous antioxidants could neutralize the excessive amount of mtROS, thereby decreasing the rate of mtROSp production and retard the aging process. However, studies have shown that the induction of an endogenous antioxidant response by exogenous agents is more effective than dietary antioxidants in counteracting oxidative stress. A study on rat brain has shown that the endogenous antioxidants, bilirubin, and biliverdin, exhibited greater antioxidant activity than alpha-tocopherol (which is a popular antioxidant supplement) in terms of preventing oxidative damage in vitro [15]. Another study using adult male houseflies suggested that the administration of exogenous antioxidants can lead to compensatory depression in endogenous antioxidant defenses [16]. Because of this, approaches, such as mitohormesis, that elicit endogenous antioxidant responses may be more effective than exogenous antioxidant ingestion in slowing down aging.

\section{Approaches to Delaying the Aging Process in Traditional Chinese Medicine}

According to Chinese medicine theory, the complete deprivation of zheng Qi leads to death [17], and accelerated aging is caused by the high rate of usage of primordial Qi, which constitutes zheng Qi together with pectoral Qi. As a congenital essence, primordial Qi cannot be replenished, but it can be spared by generating sufficient pectoral Qi derived from air and food intake to a certain extent [18]. The sustained generation of zheng Qi necessitates the presence of primordial Qi and pectoral Qi. 
From the perspective of traditional Chinese medicine, one approach to increasing lifespan and/or health span would be ensuring the balance of organ functions to safeguard the generation of pectoral Qi. With sufficient pectoral Qi generated, primordial Qi can be spared for prolonged usage rather than being utilized at a higher rate. The formation of pectoral Qi is achieved by combining air from the "Lung" and grain Qi transformed from food and water from the "Spleen" [18]. The balance between Zeng (i.e., organ) functions can have beneficial effects on the formation of pectoral Qi, which is generated by interrelated organs in the body, in particular, the "Lung" and the "Spleen".

Another approach to increasing lifespan and maintaining health is using Chinese tonifying herbs to remediate the deficiencies in Yin, Yang, Qi, and Blood in the body caused by prenatal and/or postnatal factors. According to traditional Chinese medicine theory, the imbalance of Yin and Yang is the root cause of disease, and zheng Qi is formed from the interaction between Yin (pectoral Qi) and Yang (primordial Qi). The generation of Blood from Qi is essential in nourishing internal organs in order to sustain their physiological function within the body. Hence, remediating the deficiencies in Yin, Yang, Qi, and Blood is crucial in achieving a healthy body status. To achieve this goal, functional subgroups of tonifying herbs, including Yang-invigorating, Qi-invigorating, Yin-nourishing, and Blood-enriching herbs, can be used.

Adopting a mindful lifestyle is also vital to extend the healthspan. According to the holistic theory of traditional Chinese medicine, the body as a whole is functionally connected to the mind, which implies that an imbalance of organ functions can influence the mind, and vice versa. Overly sensitive reactions or emotional fluctuations can increase the rate of primordial Qi consumption, which could in turn accelerate the rate of aging. Therefore, one key factor in maintaining physical health is ensuring an optimal balance of body-mind function. This can often be achieved by meditation, regular exercise, and quality rest periods in order to create a feeling of calm and harmony.

\section{A Unified Holistic Approach in Retarding the Aging Process}

After considering the aging process from modern medicine and traditional Chinese medicine perspectives, the generation and utilization of energy usually emerge as the primary causes of aging. Modern medicine emphasizes the role of mitochondrial dysfunction, which is determined by the rate of mtROSp in aging, while traditional Chinese medicine views aging and death as the deprivation of Qi. By integrating these two conceptual theories, the biochemical unit of Qi at the cellular level is ATP, and its generation relies on the optimal function of mitochondria. To retard the aging process, the aforementioned approach in safeguarding mitochondrial function through autophagy and/or mitophagy is consistent with the use of Yang- and Qi-invigorating Chinese tonifying herbs. Not only can Yang- and Qi-invigorating herbs increase mitochondrial membrane fluidity and a glutathione-dependent antioxidant response that can eventually 


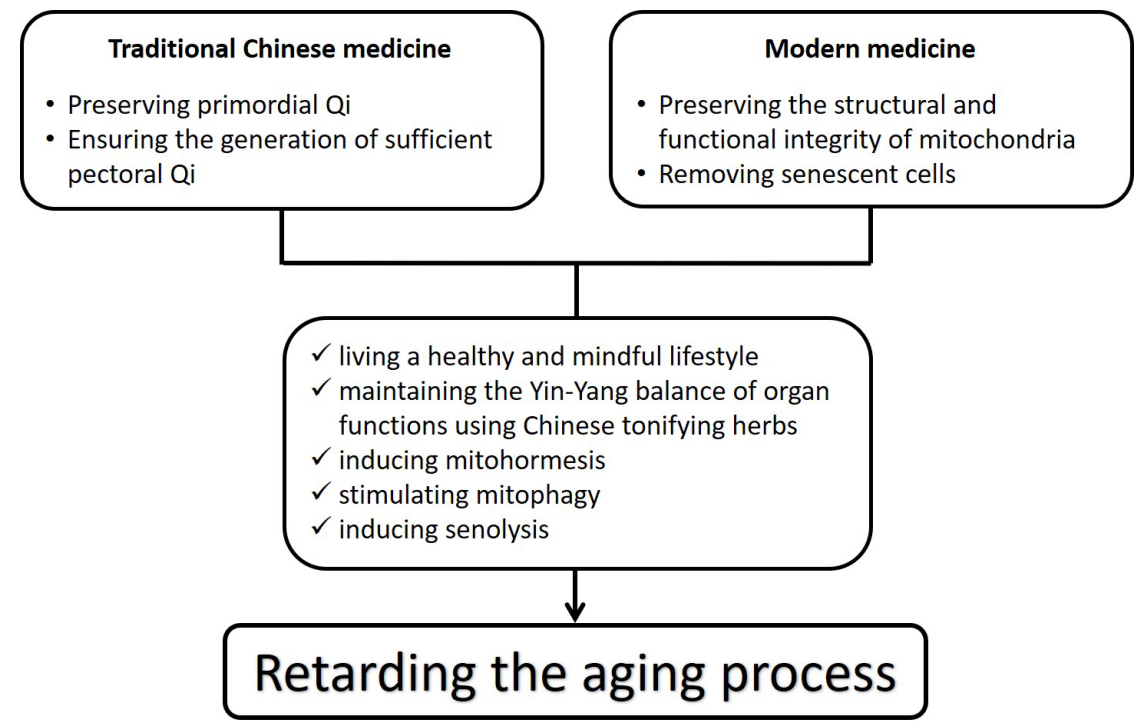

Figure 1. A unified holistic approach in retarding the aging process.

increase mitochondrial ATP generating capacity, they are also able to maintain a sustained low level of mtROS that triggers mitohormesis in vitro and ex vivo [18]. Therefore, naturally-occurring agents, such as active ingredients from Chinese tonifying herbs or their synthetic analogs, can be used to induce mitophagy and mitohormesis, with a resultant preservation of mitochondrial functional integrity. Furthermore, these herbs are also capable of maintaining an internal balance of body functions in terms of Yin, Yang, Qi, and Blood. A unifying theory of aging between modern medicine and traditional Chinese medicine could thereby culminate in an effective approach in retarding the aging process (Figure 1).

\section{Conclusion}

In conclusion, both modern medicine and traditional Chinese medicine regard the generation and utilization of energy as the primary cause of aging. Naturally-occurring or synthetic active ingredients from Chinese tonifying herbs can be used to preserve the structural and functional integrity of mitochondrial function and retard the aging process.

\section{Conflicts of Interest}

The authors declare no conflicts of interest regarding the publication of this paper.

\section{References}

[1] da Costa, J.P., Vitorino, R., Silva, G.M., Vogel, C., Duarte, A.C. and Santos, T.R. (2016) A Synopsis on Aging-Theories, Mechanisms and Future Prospects. Ageing Research Review, 29, 90-112. https://doi.org/10.1016/j.arr.2016.06.005

[2] Veith, I. (1972) The Yellow Emperor's Classic of Internal Medicine. University of California Press, Berkeley, Los Angeles, London, 15. 
[3] Barja, G. (2019) Towards a Unified Mechanistic Theory of Aging. Experimental Gerontology, 124, 110627. https://doi.org/10.1016/j.exger.2019.05.016

[4] Chabi, B., de Cararet, B.M., Chevrollier, A., Boisgard, S. and Stephen, G. (2005) Random mtDNA Deletion and Functional Consequence in Aged Human Skeletal Muscle. Biochemica Biophysica Research Communication, 332, 542-549. https://doi.org/10.1016/j.bbrc.2005.04.153

[5] Caro, A., Gómez, J., Arduini, A., González-Sánchez, M., González-García, M., Borrás, C., Viña, J., Puertas, M.J., Sastre, J. and Barja, G. (2010) Mitochondrial DNA Sequences Are Present Inside Nuclear DNA in Rat Tissues and Increase with Age. Mitochondrion, 10, 479-486. https://doi.org/10.1016/j.mito.2010.05.004

[6] Macedo, J.C., Vaz, S. and Bakker, B. (2018) FoxM1 Repression during Human Aging Leads to Mitotic Decline and Aneuploidy-Driven Full Senescence. Nature Communication, 9, Article No. 2834. https://doi.org/10.1038/s41467-018-05258-6

[7] Pamplona, R. and Barja, G. (2006) Mitochondrial Oxidative Stress, Aging and Caloric Restriction: The Protein and Methionine Connection. Biochimica et Biophysica $\operatorname{Acta}(B B A)$-Bioenergetics, 1757, 496-508. https://doi.org/10.1016/j.bbabio.2006.01.009

[8] Nakamura, S. and Yoshimori, T. (2018) Autophagy and Longevity. Molecules and Cells, 41, 65-72.

[9] Ashrafi, G. and Schwarz, T.L. (2013) The Pathways of Mitophagy for Quality Control and Clearance of Mitochondria. Cell Death and Differentiation, 20, 31-42. https://doi.org/10.1038/cdd.2012.81

[10] Bárcena, C., Mayoral, P. and Quirós, P.M. (2018) Mitohormesis, an Antiaging Paradigm. International Review of Cell and Molecular Biology, 340, 35-77. https://doi.org/10.1016/bs.ircmb.2018.05.002

[11] Yun, J. and Finkel, T. (2014) Mitohormesis. Cell Metabolism, 19, 757-766. https://doi.org/10.1016/j.cmet.2014.01.011

[12] Regulski, M.J. (2017) Cellular Senescence: What, Why, and How. Wounds. $A$ Compendium of Clinical Research and Practice, 29, 168-174.

[13] Hickson, L.J., LanghiPrata, L.G.P., Bobart, S.A., Evans, T.K., Giorgadze, N., Hashmi, S.K., Herrmann, S.M., Jensen, M.D., Jia, Q., Jordan, K.L., Kellogg, T.A., Khosla, S., Koerber, D.M., Lagnado, A.B., Lawson, D.K., LeBrasseur, N.K., Lerman, L.O., McDonald, K.M., McKenzie, T.J., Passos, J.F., Pignolo, R.J., Pirtskhalava, T., Saadiq, I.M., Schaefer, K.K., Textor, S.C., Victorelli, S.G., Volkman, T.L., Xue, A., Wentworth, M.A., Wissler Gerdes, E.O., Zhu, Y., Tchkonia, T. and Kirkland, J.L. (2019) Senolytics Decrease Senescent Cells in Humans: Preliminary Report from a Clinical trial of Dasatinib plus Quercetin in Individuals with Diabetic Kidney Disease. Ebiomedicine, 47, 446-456. https://doi.org/10.1016/j.ebiom.2019.08.069

[14] Li, W., Qin, L., Feng, R., Hu, G., Sun, H., He, Y. and Zhang, R. (2019) Emerging Senolytic Agents from Natural Products. Mechanism of Ageing and Development, 181, 1-6. https://doi.org/10.1016/j.mad.2019.05.001

[15] Mancuso, C., Barone, E., Guido, P., Miceli, F., Di Domenico, F., Perluigi, M., Santangelo, R. and Preziosi, P. (2012) Inhibition of Lipid Peroxidation and Protein Oxidation by Endogenous and Exogenous Antioxidants in Rat Brain Microsomes in Vitro. Neuroscience Letters, 518, 101-105. https://doi.org/10.1016/j.neulet.2012.04.062

[16] Sohal, R.S., Allen, R.G., Farmer, K.J., Newton, R.K. and Toy, P.L. (1985) Effects of Exogenous Antioxidants on the Levels of Endogenous Antioxidants, Lipid-Soluble Fluorescent Material and Life Span in the Housefly, Musca domestica. Mechanisms 
of Aging and Development, 31, 329-336.

https://doi.org/10.1016/0047-6374(85)90098-3

[17] Zhang, D. and Wu, X. (1991) Qi, Blood, Body Fluid, Essence of Life and Spirit. In: Liu, Y., Ed., The Basic Knowledge of Traditional Chinese Medicine, Hai Feng Publishing Co., Hong Kong, Chapter 5, 49-53.

[18] Leong, P.K., Chen, J. and Ko, K.M. (2018) Development of Chinese Herbal Health Products for the Prevention of Aging Associated Diseases. In: Mandal, S.C., et al., Eds., Natural Products and Drug Discovery, Elsevier Ltd., Netherlands, Chapter 4, 73-104. https://doi.org/10.1016/B978-0-08-102081-4.00004-6 